\title{
A ATRATIVIDADE DO TRANSPORTE PÚBLICO PARA AS PESSOAS COM LIMITAÇÕES FÍSICAS E OS COMPORTAMENTOS NA MOBILIDADE URBANA
}

\author{
SOUZA, Raphael Freitas (1); \\ MARTINS, Laura Bezerra (2) \\ (1) Universidade Federal de Pernambuco, Mestrando em Design \\ e-mail: raphael.freitassouza@ufpe.br \\ (2) Universidade Federal de Pernambuco, Doutora \\ e-mail: bmartins.laura@gmail.com
}

\begin{abstract}
RESUMO
Este artigo apresenta o estado da arte sobre o comportamento de usuários, com e sem limitações físicas, no transporte público. Por meio de uma Revisão Sistemática da Literatura, identificou-se as abordagens, os métodos e técnicas dos estudos, e verificou-se que os fatores de qualidade do transporte público influenciam comportamentos de mobilidade e percepções subsequentes do sistema. As pessoas com limitações físicas, além da segurança, confiabilidade, frequência, pontualidade e a qualidade da informação, tendem a se preocupar com questões como distâncias dos deslocamentos complementares, facilidade do acesso, disponibilidade de veículos adaptados, e condições e lotação das calçadas, terminais e veículos.
\end{abstract}

Palavras chave: Comportamentos na Mobilidade Urbana; Transporte público; Deficiência física.

\begin{abstract}
This paper shows the state of the art of the studies about user behaviors, with and without physical impairments in public transport. A systematic review of the literature identified the mainly approaches, methods, techniques of the studies and, verified that the quality factors of the public transport affects the mobility behaviors and the subsequent perceptions of the system. In addition to security, safety, reability, frequency, punctuality and the information quality, the physical impairment people tend to worry about questions as distances of complementary displacements, ease access, availability of adapted vehicle, and conditions, occupancy of the sidewalks, terminals and vehicles.
\end{abstract}

Keywords: Urban Mobility Behaviors; Public Transport; Physical Impairment.

\section{INTRODUÇÃO}

Nos últimos anos, a queda da atratividade e a baixa percepção da qualidade dos sistemas de transporte público, têm reduzido gradativamente o número de usuários. Dessa forma, torna-se relevante a identificação dos fatores de qualidade, bem como o comportamento dos usuários em relação a eles, para que se possa oferecer soluções que tornem a utilização do transporte mais confiável e atrativa. Dentro dessas questões relativas à redução da qualidade percebida, uma das causas envolvidas é a insuficiência dos sistemas de informação ao usuário, de prover orientação, sendo que muitas pessoas, portanto, deixam 


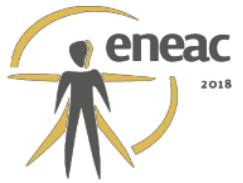

de utilizar o transporte apenas devido à falta de informação, evitando assim se expor a situações inseguras e estressantes (MINISTÉRIO DAS CIDADES, 2008; WRIGHT, 2001).

Para o caso das pessoas que possuem limitações físicas, acredita-se que somados aos fatores que afetam a maioria dos usuários, possam existir outros devido às suas condições individuais e a falta de adaptabilidade dos ambientes envolvidos no contexto dos deslocamentos pelo transporte público, sendo necessários mais estudos que problematizem a mobilidade e atratividade do transporte público para este tipo de usuário.

A pesquisa busca responder a seguinte pergunta problema: Quais fatores afetam a mobilidade urbana por transporte público e que informações são importantes para que 0 usuário com limitação física se sinta mais confiante e atraído em utilizá-lo?

Tendo em vista que as pessoas com limitações físicas, além de suas questões específicas sofrem também dos problemas que são comuns aos usuários sem limitações, o objetivo geral dessa presente pesquisa foi o de investigar o estado da arte dos estudos sobre comportamentos de mobilidade urbana por transporte público dos dois grupos.

Entre os objetivos específicos procurou-se: identificar as abordagens utilizadas nos estudos selecionados; mapear os métodos e técnicas empregados; e apontar os fatores que afetam tanto a preferência pelo transporte público, quanto a escolha por rotas e os comportamentos nos deslocamentos.

Levando em consideração a dispersão de estudos a respeito dos objetos analisados, o presente artigo procura contribuir com sua sistematização por meio de revisão sistemática da literatura (RSL). Esse estudo teve como relevância, guiar futuras pesquisas, com a abordagem centrada no usuário, sobre a mobilidade urbana por transporte público para pessoas com deficiência física-motora.

\section{MATERIAL E MÉTODO}

Trata-se de uma pesquisa bibliográfica de abordagem descritiva pautada na Revisão Sistemática da Literatura, a qual foi realizada por meio de consultas no portal de periódicos CAPES. Optou-se por essa metodologia, por reduzir ao máximo os vieses da pesquisa e possibilitar uma análise mais objetiva e sistematizada dos resultados, facilitando uma síntese conclusiva sobre o assunto (SAMPAIO \& MANCINI, 2007). Após os testes de sintaxe e semântica, foram definidas as estratégias de busca, que estão descritas na Tabela 1 , juntamente com os objetivos e o número de artigos selecionados. 


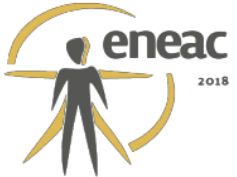

Tabela 1: Resultados da busca e seleção de artigos considerados na pesquisa

\begin{tabular}{|c|c|c|c|c|c|c|c|}
\hline \multirow{4}{*}{1} & TÓPICOS & & ESTRATÉGIAS DE BUSCA & & 1" Sel. & $2^{\text {a }}$ Sel. & OBJETIVOS \\
\hline & \multirow{3}{*}{$\begin{array}{l}\text { COMPORTAMENTOS } \\
\text { NA MOBILIDADE } \\
\text { URBANA }\end{array}$} & 1.1 & $\begin{array}{l}\text { "urban mobility" AND behavior AND "public } \\
\text { transport" }\end{array}$ & 17 & 5 & 3 & \multirow{4}{*}{$\begin{array}{l}\text { Comportamentos da } \\
\text { mobilidade urbana no } \\
\text { contexto de transporte } \\
\text { público do ponto de vista do } \\
\text { usuário. Estudos com } \\
\text { usuários - Estado da arte e } \\
\text { metodologias empregadas }\end{array}$} \\
\hline & & 1.2 & $\begin{array}{l}\text { "public transport" AND orientation AND } \\
\text { wayfinding }\end{array}$ & 58 & 14 & 13 & \\
\hline & & 1.3 & $\begin{array}{l}\text { urban mobility AND wayfinding AND } \\
\text { behavior AND "public transport" }\end{array}$ & 7 & 3 & 0 & \\
\hline 2 & $\begin{array}{l}\text { MOBILIDADE } \\
\text { ACESSIVEL - } \\
\text { ESTUDOS COM } \\
\text { USUÁRIO }\end{array}$ & 2.1 & $\begin{array}{l}\text { Accessibility AND physical AND (impaired } \\
\text { OR disabled) AND "public transport" NOT } \\
\text { policy NOT (medice OR medical) }\end{array}$ & 70 & 20 & 11 & \\
\hline \multirow[t]{2}{*}{3} & $\begin{array}{l}\text { INFORMAÇÃO DO } \\
\text { TRANSPORTE } \\
\text { PÚBLICO }\end{array}$ & 3.1 & $\begin{array}{l}\text { information AND "user information" AND } \\
\text { "information system" AND "public transport" }\end{array}$ & 43 & 14 & 3 & $\begin{array}{l}\text { Sist. de informação e o } \\
\text { usuário do transporte público. } \\
\text { Estudos com usuários - } \\
\text { Estado da arte e } \\
\text { metodologias empregadas }\end{array}$ \\
\hline & TOTAL & & & 195 & 56 & 30 & \\
\hline
\end{tabular}

Os resultados foram filtrados por publicações no período de 2007-2017, nos idiomas Português, Inglês e Espanhol e revisados por pares. Para se organizar os dados, foram montadas planilhas no Microsoft Excel, contendo colunas de título, autores, palavras-chave/ assuntos, ano de publicação, resumos, motivo de relevância ou descarte e metodologia, e colocados separados pelos tópicos e estratégias de buscas. Na primeira seleção, a partir da leitura dos títulos e resumos, foram selecionados aqueles que se relacionavam ao comportamento de usuários no transporte público, os deslocamentos a pé e os fatores externos envolvidos. Foram definidos como critérios de inclusão:

- Estudos com usuários;

- Impactos das limitações físicas na motivação no Transporte Público;

- Fatores de atratividade e qualidade do transporte público;

- Impacto da informação sobre os comportamentos de deslocamentos;

- Transporte urbano/ semiurbano e deslocamentos a pé;

- Metodologias de captação de comportamentos de usuários em relação aos deslocamentos pelo transporte público;

- Características de orientação e informação no transporte público;

- Adultos acima de 18 anos sem limitações intelectuais.

Foram excluídos os artigos de revisão da literatura, aqueles que não envolviam o público mencionado acima, ou focavam em outros tipos de limitações e deficiências (visual, auditiva, intelectual, etc) e também aqueles que não consistiam em estudos relativos à comportamentos de usuários. Também se descartou pesquisas que focavam em políticas públicas urbanas, questões médicas, de exclusão social e tecnologias. $O$ número apresentado na tabela dos selecionados se considera também a eliminação de duplicatas.

Após a primeira seleção, os artigos foram acessados e lidos com o foco nas seções de metodologia, resultados e conclusões. Foram descartados, aqueles que com a leitura, não tinham relevância para a pesquisa devido aos critérios já mencionados. Alguns dos selecionados na primeira etapa também foram descartados por não possuírem acesso pelo portal CAPES. Ao todo, foram utilizados para a análise 30 artigos. 


\section{remes $^{-1}$}

\section{ANÁLISE E DISCUSSÃO DE RESULTADOS}

Com a leitura dos artigos selecionados, as próximas etapas foram: identificar as abordagens e metodologias utilizadas, e por fim, os principais resultados e conclusões relativas aos fatores que afetam tanto a preferência pelo transporte público, quanto a escolha por rotas e os comportamentos nos deslocamentos.

\subsection{Abordagens}

Com a identificação das abordagens de cada artigo, foram identificadas as principais temáticas. Os artigos então foram agrupados de acordo com elas. Sendo assim, os estudos abordaram os seguintes temas:

- Fatores que afetam escolha do modo de transporte e das rotas - Resultados que apontam a relação de questões das experiências dos usuários, incluindo a qualidade e as características das informações e dos mapas esquemáticos, sobre a escolha modal, e o processo decisório de rotas.

- Percepção da qualidade e satisfação do sistema de transportes - Estudos onde foram feitas avaliações e a identificação dos fatores de qualidade que possuem mais impacto na percepção e comportamento dos usuários.

- Sistemas de informação ao usuário no transporte público - Estudos que abordam as informações mais importantes para um deslocamento com maior confiança e menos estresse.

- Mapas esquemáticos e mapas cognitivos - Como extensão do sistema de informações, são estudos que mostram a interpretação dos mapas sobre o comportamento dos usuários e as representações mentais.

- Circulação de pessoas - Estudos relativos à comportamentos de mobilidade em ambientes com grande concentração de pessoas, e situações de emergência.

- Tecnologias para Wayfinding - Estudos que citam tecnologias de apoio à orientação espacial e mobilidade, incluindo de pessoas com deficiência física, como sites e mapas colaborativos e aplicativos.

- Acessibilidade e Usabilidade - Estudos que tratam dos deslocamentos no transporte público e complementares de cadeirantes e idosos, e as percepções da acessibilidade pelos usuários.

\subsection{Métodos e técnicas utilizadas}

Em relação às técnicas empregadas foram utilizados questionários, entrevistas, grupos de foco/ workshops, experimentos de simulações e cenários, testes de usabilidade, observação, investigação documental e prototipagem. Em muitas das pesquisas, foi verificado uma combinação de mais de uma técnica de forma complementar. A seguir serão descritas as aplicações das técnicas utilizadas.

\section{a) Questionários}

A técnica mais utilizada foi o questionário. Uma aplicação largamente utilizada nos estudos foi de escalas de avaliação, como a escala Likert, dada uma lista de fatores relacionados à percepção da qualidade do sistema de transportes e da necessidade da informação (LOIS, MONZỎN \& HERNANDÉZ, 2016; MACHADO-LEÓN ET AL. 2017). A partir das médias de 
avaliações, em algumas pesquisas foram realizadas análises estatísticas com o objetivo de mensurar e comparar os valores, identificando fatores que mais impactavam na percepção da qualidade do sistema e da necessidade da informação (GRISON, GYAWLINCK, BURKHARDT, 2016; LOIS, MONZÓN \& HERNANDÉZ 2017; TYRINOPOULOS \&

ANTONIOU, 2012; STOPKA, BARTUSKA, KAMPF, 2015). Em outras como, Grotenhuis, Wiegmans \& Rietveld, (2007) e Tuominen et al., (2007), adotou-se a estratégia de analisar os resultados, por classificação de grupos por gênero, idade, atividade, localização, tipo de área residencial e padrões de mobilidade similares, e assim identificar perfis de usuários.

Diante desse objetivo citado, no estudo de Tyrinopoulos \& Antoniou (2012), a aplicação de questionários, serviu para medir o grau de fatores que afetam a escolha modal, e outros fatores que desencorajam o uso de um determinado tipo de transporte. Grotenhuis, Wiegmans, Rietveld (2007), também aborda a qualidade da informação, na viagem multimodal, utilizando a escala Likert para investigar as informações consideradas mais importantes em três etapas das viagens: Pré-viagem; pré-embarque; e a bordo. Outras pesquisas como Jarzemskis \& Jarzemskiene (2017), também envolveram preferências e comportamentos de mobilidade por modais (transporte público, transporte fretado/escolar, bicicleta, automóvel, e deslocamentos a pé) e no questionário foram abordadas questões, como modal de transporte, duração média das viagens, origem e destino, e critérios de satisfação e expectativas na mobilidade para cada modal.

Percebe-se que a maioria das pesquisas adotou o questionário com a proposta de utilizar os resultados para levantamento de dados, excluindo Madl et al. (2016), que usou a ferramenta para fins de recrutamento. Além das finalidades descritas, os questionários cumpriram a finalidade de levantar também questões socioeconômicas, demográficas e de contexto dos deslocamentos e usuários. O conteúdo em geral foi formado de perguntas objetivas, no entanto em Ahmad (2015), junto com as escalas Likert, foi pedido aos participantes que justificassem suas avaliações.

O modo da divulgação envolveu o meio on-line, como e-mail, jornais internos universitários, telefone, mas também estratégias diferenciadas, como em Lois, Monzón \& Hernandéz (2017), que os pesquisadores, nos terminais, entregaram pessoalmente cartões contendo um link para o questionário. Em Ahmad (2015) já foi usada a técnica do Snowball, onde os indivíduos selecionados convidaram amigos e conhecidos para participarem dos estudos.

\section{b) Entrevistas}

As entrevistas foram utilizadas para um entendimento mais completo, na maioria das vezes de forma complementar às outras técnicas, abordando experiências e opiniões dos usuários. Em Ahmad (2015), foi investigado a severidade das deficiências dos participantes, após a aplicação do questionário. Em May et al. (2014), a entrevista sucedeu um teste de usabilidade. Na pesquisa de Grison, Gyawlinck, Burkhardt (2016), o propósito foi investigar a satisfação ou insatisfação relacionada à escolha de rotas do transporte público, sendo pedido aos participantes para reportarem os incidentes mais marcantes ocorridos por eles no passado, de maneira complementar a um questionário com escala Likert. Já em Bromley, Matthews \& Thomas (2007) a finalidade foi investigar e reavaliar a acessibilidade atual do centro da cidade, com o foco nos deslocamentos para a atividade de compras. Diferentemente das pesquisas citadas, o estudo de Lois, Monzón \& Hernandéz (2017), apenas utilizou a entrevista como um primeiro e breve contato com os participantes, antecedendo uma aplicação de um questionário.

\section{c) Grupos de Foco e Workshops}

Os grupos de foco relatados nas pesquisas, tiveram o objetivo de levantar percepções individuais e necessidades informacionais de pessoas com deficiência. Em Eichhorn et al. (2008), foi realizado um workshop com grupos de usuários de pessoas com deficiência, mas também com representantes de organizações que tratam assuntos com deficiência, 


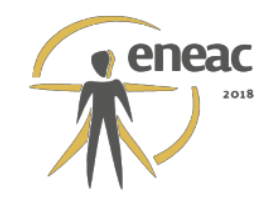

agências de viagens especializadas, operadores de turismo, para discutirem a acessibilidade em sistemas de comunicação de informações turísticas baseado em fatores como detalhamento, confiabilidade da informação, fontes apropriadas, ferramentas de comunicação e serviços orientados ao usuário.

Já em Westerheim, Haugset \& Natvig (2007), foi organizado um workshop com representantes de grupos de pessoas com deficiências para discutirem a partir de determinados cenários como: Chegar em uma parada de ônibus normal e entrar no veículo; utilizar ônibus, metrô ou trem para chegar em um terminal e acessar o trem expresso para o aeroporto; sair do trem expresso e transferir para um voo. O objetivo foi levantar que tipo de informação é necessária para os passageiros, de acordo com cada tipo de deficiência. Houve uma fase posterior na pesquisa, que o pesquisador e esses representantes de pessoas com deficiência, físicas, visual e auditiva, visitaram esses terminais e continuaram a discussão iniciada no workshop.

\section{d) Simulações e Cenários e testes de usabilidade}

Essas técnicas foram utilizadas com o propósito de investigar comportamentos dos usuários dado uma determinada situação. Haghani, Sarvi (2017a), Haghani, Sarvi (2017b) e Haghani et al. (2016) foi descrito experimentos que simulavam evacuações.

Em Madl et al. (2016), para investigar a construção dos mapas cognitivos, foi primeiro pedido aos participantes para desenharem mapas esquemáticos com alguns dos marcos de referência das cidades que habitavam. Em seguida, um segundo experimento envolveu que os participantes arrastassem alguns marcos de referência, em uma tela virtual interativa estabelecendo o posicionamento relativo e determinando o tempo para se deslocar entre eles.

No estudo de May et al. (2014), foi realizado um teste de um sistema de mapas colaborativos (mashup) com dois grupos: participantes com limitação física e pais de crianças em carrinhos de bebê. Foi pedido aos participantes que realizassem as tarefas dos testes, para ser investigado que tipo de informações esses grupos mais necessitavam. A cada atividade que executassem eles deveriam comentar em voz alta o que realizavam, uma técnica que é chamada de think aloud.

Em Westerheim, Haugset \& Natvig (2007), a criação de cenários, ocorreu para se levantar discussões dada uma determinada situação, como foi explicado no tópico anterior. Já na pesquisa de Jacob et al. (2012), foram investigados os efeitos das tecnologias táteis no processo de Wayfinding, através de testes com smartphones, com um tipo de aplicativo que emitia alarmes por vibração e descrições de texto, assim que o participante se aproximava de um marco de referência, como uma forma de feedback. O objetivo foi de verificar se o recurso auxiliava a navegação espacial quando o usuário realiza uma outra atividade simultânea, como conversar, e se melhorava a memorização espacial, se comparado com um sistema visual.

\section{e) Observação: Fotos, filmagens, passeio acompanhado, sintaxe espacial}

As técnicas de observação tiveram como objetivo analisar as interações dos usuários com o ambiente e a dinâmica da circulação de pessoas. Foram utilizadas fotografias e observação das características ambientais de acessibilidade em Meshur (2013); filmagens em Haghani, Sarvi (2017a), Haghani, Sarvi (2017b) e Haghani et al. (2016) de simulações para evacuações; e a instalação de câmeras em veículos para se estudar a usabilidade de ônibus piso-baixo em Hwangbo et al. (2015).

No estudo de Ludwig et al. (2013), foi realizado um passeio acompanhado de forma simultânea a uma análise tarefa da utilização de um aplicativo de navegação espacial para pedestres e usuários do transporte público, sendo observado o tempo de execução da tarefa, o número de eventos de desorientação e o número de olhadas para a tela. Por 


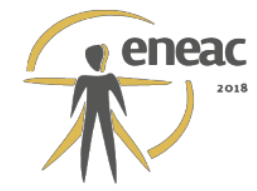

último, Van Der Hoeven \& Van Nes (2014) descrevem o uso de sintaxe espacial para avaliar o fluxo de pessoas em estações de metrô, com o objetivo de propor soluções que podem melhorar a orientação nessa situação.

\section{f) Investigação Documental}

A técnica foi utilizada em dois estudos. Na pesquisa de Jarzemskis \& Jarzemskiene (2017) foi feito uma investigação de documentos oficiais (como planos de ação e mobilidade e políticas urbanas) que mostravam as diferenças nas listas de atributos da qualidade de transporte na União Europeia ao longo dos anos. Dado esse levantamento foi realizado uma comparação com os fatores de qualidade percebidos pelos usuários - os habitantes de Siauliai (Lituânia) - através da aplicação de um questionário. Já no estudo de Westerheim, Haugset \& Natvig (2007), a investigação se baseou em documentos para se avaliar o status do trabalho de acessibilidade na Noruega.

\section{g) Prototipagem}

Nos estudos encontrados, foram desenvolvidos protótipos com propostas de sistemas e aplicativos de navegação espacial com objetivo de auxiliar os deslocamentos e orientação inclusive de usuários que possuem ou não deficiência.

$\mathrm{Na}$ pesquisa de Jacob et al. (2012), o protótipo consistia em alertas vibratórios em smartphones que auxiliavam a navegação espacial, como já apresentado. Com algumas similaridades, Rodriguez-sanchez \& Martinez-romo (2017), descreveram a plataforma Gawa, desenvolvida a partir de conceitos de Design Universal e com recursos como feedback tátil, vibratório e de linguagem de sinais. Uma outra solução proposta foi a apresentada por Ludwig et al. (2013), que consistia em um sistema de guia, baseado numa representação virtual e tridimensional de marcos de referência, sendo exibidas algumas das características principais, cor, tamanho e localização, auxiliando nos deslocamentos porta-a-porta, tendo abrangência tanto de ambientes abertos, quanto fechados.

Já com o foco na informação compartilhada, May et al. (2014) cita o desenvolvimento de uma proposta de sistema alimentado por voluntários, que poderiam informar sobre a facilidade de acesso de pontos de interesse e as ligações de transporte.

\subsection{Fatores que afetam as preferências, a escolha de rotas e os comportamentos nos deslocamentos.}

Os artigos analisados nessa revisão apontam fatores que afetam a escolha modal e de rotas, e os comportamentos de mobilidade, inclusive a percepção da qualidade do sistema de transportes. Também foram levantadas nos resultados, as informações necessárias para a maior confiabilidade no transporte público, sendo abordado aquelas que atendem as necessidades tanto do público que possui limitações físicas na mobilidade, quanto do que não possui nenhum tipo de limitação.

\section{a) Qualidade do transporte público}

Os principais fatores apontados ligados à percepção de qualidade do transporte público foram (AHMAD, 2015; JARZEMSKIS \& JARZEMSKIENE, 2017; GRISON, GYAWLINCK, BURKHARDT, 2016; HERNANDEZ, MONZÓN \& LOIS, 2016; LIN ET AL., 2014; MACHADO-LEÓN ET AL. 2017; MONZON, HERNANDEZ, CASCAJO, 2013; SUSILO, CATS, 2014; TYRINOPOULOS \& ANTONIOU, 2012):

- Boa Frequência e pontualidade;

- Facilidade de acesso e de transferências entre linhas e modais;

- Acessibilidade; 


\section{ineac}

- Preço acessível;

- Conforto e lotação;

- Segurança;

- Proximidade das estações com áreas comerciais;

- Qualidade da informação.

No entanto, vários autores destacam que esses fatores podem variar e tendem a ter pesos diferentes de acordo com os grupos de usuários, a motivação de uso e contextos distintos de cada localidade. (GRISON, GYAWLINCK, BURKHARDT, 2016; GROTENHUIS, WIEGMANS, RIETVELD, 2007; SUSILO, CATS, 2014; WESTERHEIM, HAUGSET \& NATVIG, 2007). Para as pessoas com deficiência as características que possuem a maior relevância nos seus deslocamentos são (AHMAD, 2015, WESTERHEIM, HAUGSET \& NATVIG, 2007):

- Acessibilidade;

- A disponibilidade de transporte acessível;

- Distância da origem para estação e conexões entre linhas e modais;

- Preço acessíveis;

- Arranjo de assentos;

- Segurança;

- Qualidade do serviço e facilidades.

Como os deslocamentos a pé são considerados complementares ao transporte público, Jarzemskis \& Jarzemskiene (2017), cita alguns dos fatores que os usuários levam em consideração: lluminação, distâncias, boa rede de trilhas para pedestres, segurança da infraestrutura e segurança pública.

Não restringindo apenas aos deslocamentos a pé, algumas pesquisas citam o peso que segurança pública recebe em alguns locais, especialmente para as mulheres (AHMAD, 2015; HERNANDEZ, MONZÓN \& LOIS, 2016; JARZEMSKIS \& JARZEMSKIENE, 2017; LI

ET AL., 2017; MACHADO-LEÓN ET AL. 2017). É citado que em locais como o Paquistão, as mulheres especialmente as cadeirantes são particularmente expostas à violência, pois muitas vezes são marginalizadas e consideradas incapazes, e assim o fator segurança ganha um grande destaque nos comportamentos de mobilidade de tal país (AHMAD, 2015).

Outro ponto levantado é que as primeiras experiências com uma rota ou sistema de transporte podem ter um papel decisivo nos comportamentos e percepções das posteriores, em relação à qualidade (SHHMITT, CURRIE \& DELBOSC, 2013; SUSILO \& CATS, 2014). Por fim, os fatores como qualidade ambiental e design, embora possuam importância menor do que os citados, ainda sim influenciam significantemente a qualidade percebida (HERNANDEZ, MONZÓN \& LOIS, 2016; SUSILO \& CATS, 2014).

Algumas pesquisas também citam os fatores que desencorajam o uso do transporte. Além de questões como segurança para as pessoas com limitação física na mobilidade, os problemas mais relevantes são (AHMAD, 2015; BROMLEY, MATTHEWS \& THOMAS, 2007).

- Veículos cheios;

- Disponibilidade baixa de veículos que possam acomodá-los bem;

- Terminais em mau estado; 


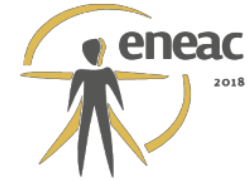

- Calçadas obstruídas;

- Atendimento ruim de motoristas e operadores do transporte;

- Dificuldade de acesso e ausência de rampas;

- Ausência de locais para travessia segura.

Devido a isso, alguns grupos de cadeirantes, idosos, e pessoas com deficiências mais severas, são menos propensas a se deslocarem, ou adaptam seus hábitos de deslocamento em horários mais vazios (BROMLEY, MATTHEWS \& THOMAS; MAY ET AL., 2014). Dessa forma torna-se ainda mais relevante identificar esses problemas para os passageiros terem acesso às informações relativas às condições dos espaços e serviços que irão utilizar, e conseguirem se deslocar de maneira mais satisfatória.

\section{b) A informação no transporte público}

Conforme mencionado no tópico anterior, a informação pode otimizar as viagens e a sua qualidade corresponde a um importante fator de atratividade do transporte público, colaborando para a sua confiabilidade e imagem (HERNANDEZ, MONZÓN \& LOIS, 2016; MONZON, HERNANDEZ \& CASCAJO, 2013). Da mesma forma que foi citado no tópico dos fatores de qualidade, a demanda por informações varia entre os grupos de usuários e contextos. Os idosos necessitam de mais informações do que os jovens, em especial para evitar esforço físico desnecessário, sendo apropriado informações como tempo de viagem, distâncias percorridas a pé (GROTENHUIS, WIEGMANS, RIETVELD, 2007). May et al. (2014), aborda que pessoas com deficiência possuem uma grande demanda pela informação pré-viagem e a informação em tempo real, enquanto outros usuários que possuem limitações temporárias na mobilidade, como pais que precisam se deslocar com carrinho de bebê, tentem a preferir informações com um contexto. Além disso, o tipo das informações consideradas mais importantes pode variar ao longo das etapas dos deslocamentos.

Grotenhuis, Wiegmans, Rietveld (2007) dentro do contexto Holandês de viagens intermodais, dividem as viagens em três estágios: Pré-viagem; pré-embarque; e a bordo. No primeiro - a pré-viagem - as informações avaliadas como mais importantes são: mapa com as rotas, trocas, conexões e paradas; apresentação das rotas mais rápidas, mas também das alternativas; tempo total de viagem; horários de partidas; tempo de espera; alterações e atrasos; dicas de rotas; rotas a pé e tempo de caminhada até as paradas e estações. No segundo - o pré-embarque - também são consideradas importantes a presença de quadro de horários; apresentação das rotas mais rápidas e alternativas; principais trocas; tempo de espera; mas também as informações em tempo real. Já no terceiro - a bordo - as mais importantes envolvem as conexões, alterações informações em tempo real, e tempo de viagem remanescente.

\section{CONSIDERAÇÕES FINAIS}

Dentro do objetivo de se levantar o estado da arte dos estudos de comportamento na mobilidade urbana pelo transporte público, foram identificadas diferentes abordagens, métodos e técnicas, e comportamentos, percepções e preferências de mobilidade. Ao comparar as pesquisas, houve concordâncias em muitos de seus aspectos, como as similaridades entre as temáticas e no uso dos métodos e técnicas, sendo o motivo de se agrupar os estudos por esses critérios. Em relação às temáticas, essa identificação pode contribuir para futuros estudos, por se saber o que já foi estudado em cada um desses aspectos comportamentais da mobilidade. Já em relação aos métodos, percebendo que muitos deles são comuns a estudos tanto do público sem deficiência, quanto do que possui limitação física, saber quais os mais recorrentes, o por quê foram utilizados, e o tipo de 


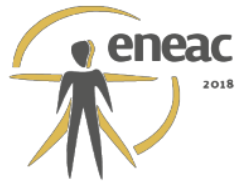

resultados que levantaram, pode auxiliar na metodologia de futuras pesquisas, dados seus objetivos.

Diante dos resultados obtidos, pôde-se verificar que os fatores de qualidade, que incluem a informação oferecida, influenciam os comportamentos de mobilidade e as percepções subsequentes do sistema. Sendo assim torna-se relevante utilizar esses dados, como base para o desenvolvimento de sistemas de informação do transporte público, permitindo ao usuário planejar seus deslocamentos com um grau maior de confiabilidade, satisfação e segurança.

Identificar as dificuldades e percepções de qualidade do sistema de transporte pelas pessoas com deficiência física e desenvolver os sistemas de informação considerando tais fatores, podem fazer com que utilizem mais o transporte público. Verificou-se que embora essas pessoas possuam algumas percepções de qualidade do sistema de transporte similares as daquelas sem deficiência, existem determinados fatores que recebem pesos diferentes. Elas também necessitam de uma demanda de informações mais completa, abrangendo questões específicas que não são tão relevantes para os demais públicos, como as condições das calçadas.

No entanto, é importante destacar que muitos desses resultados apresentados por esses artigos não podem ser tidos como universais devido às realidades e contextos diferentes de cada localidade e de cada grupo de usuários estudado. Em algumas localidades por exemplo, existem problemas sociais sérios como segurança pública, assédio e a violência contra as mulheres, além do aspecto de considerar mulheres com deficiência incapazes e marginalizadas, enquanto em outras além de não serem colocados como relevante tais questões, a rede de transportes é melhor resolvida e abrangente, com problemas menores.

Sendo assim torna-se essencial ao serem desenvolvidas estratégias para atuar na atratividade do transporte público e nos sistemas de informação, uma investigação do contexto de determinadas localidades, com uma abordagem centrada nos respectivos grupos de usuários.

\section{REFERÊNCIAS BIBLIOGRÁFICAS}

AHMAD, M. Independent-mobility rights and the state of public transport accessibility for disabled people: Evidence from southern Punjab in Pakistan. Administration and Society, Blacksburg, v. 47, n. 2, p. 197-213, mar. 2015.

BROMLEY, R.D.F., MATTHEWS, D.L., THOMAS, C.J. City centre accessibility for wheelchair users: the consumer perspective and the planning implications. Cities, v. 24, n.3. p. 229-241, jun. 2007.

EICHHORN, V., MILLER, G., MICHOPOULOU, E., \& BUHALIS, D. Enabling access to tourism through information schemes? Annals of Tourism Research, v. 35, n. 1, p. $189-210,2008$.

GRISON, E., GYSELINCK, V., \& BURKHARDT, J. M. Exploring Factors Related to Users' Experience of Public Transport Route Choice: Influence of Context and Users profiles. Cognition, Technology and Work, v. 18, n. 2, p. 287-301, 2016.

GROTENHUIS, J.-W., B. W. WIEGMANS, and P. RIETVELD (2007). The Desired Quality of Integrated Multimodal Travel Information in Public Transport: Customer Needs for Time and Effort Savings. Transport Policy, v. 14, n. 1, p. 27-38, 2007.

GUO, Z. Mind the Map! The Impact of Transit Maps on Path Choice in Public Transit. Transportation Research Part A, v. 45, p. 625-639, 2011.

HAGHANI M, SARVI M. Following the crowd or avoiding it? Empirical investigation of imitative 


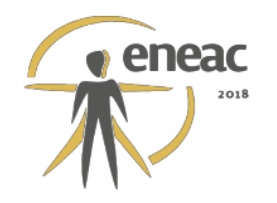

behaviour in emergency escape of human crowds. Animal Behaviour. v. 124, p. 47-56, 2017a

How perception of peer behaviour influences escape decision making: The role of individual differences. Journal of Environmental Psychology. v. 51, p. 141-157, 2017b

HAGHANI M, SARVI M, SHAHHOSEINI Z, Boltes M. How Simple Hypothetical-Choice Experiments Can Be Utilized to Learn Humans' Navigational Escape Decisions in Emergencies. PLOS One, v. 11, n. 11, p. $1-24,2016$

HWANGBO, Hwan, JIYEON Kim, SUNWOONG Kim, and Yong Ji. 2012. "Toward Universal Design in Public Transportation Systems: An Analysis of Low Floor Bus Passenger Behavior with Video Observations." Human Factors and Ergonomics in Manufacturing \& Service Industries, v. 25 n. 2, p. 183-197, 2017.

JACOB, R.; WINSTANLEY, A.; TOGHER, N.; ROCHE, R.; MOONEY, P. Pedestrian Navigation Using the Sense of Touch. Computers, Environment and Urban Systems, v. 36, p. 513-525, 2012.

JARZEMSKIS, A., JARZEMSKIENE, I. Evolution of Traveller Experience Quality Perception in European Level Policy Documents and the Case Study for Siauliai. Transport and Telecommunication Journal, Riga, v. 18, n. 3, p. 220-230, 2017.

LI, Z.; HENSHER, D.A. Crowding and public transport: A review of willingness to pay evidence and its relevance in project appraisal. Transport Policy, v. 18, n. 6, p. 880-887, 2011

LIN, T., J. XIA, T. P. ROBINSON, K. G. GOULIAS, R. L. CHURCH, D. OLARU, J. TAPIN, and R. HAN. 2014. Spatial Analysis of Access to and Accessibility Surrounding Train Stations: A Case Study of Accessibility for the Elderly in Perth, Western Australia. Journal of Transport Geography, v. 39, p. 111-120, 2014.

LOIS, D., MONZÓN, A., HERNÁNDEZ, S. Analysis of satisfaction factors at urban transport interchanges: Measuring travelers' attitudes to information, security and waiting. Transport Policy, 2017.

LUDWIG B, BIENK S, KATTENBECK M, MÜLLER M, Ohm C, EINMAL M, GLASER T, HackI M, Oreskovich M, Schubart L. Do you recognize that building's façade? Künstliche Intelligenz, v. 27, n. 3, p. 241-246, 2013

MACHADO-LEÓN, José Luis \& de Oña, Rocío \& Baouni, Tahar \& de Oña, Juan. Railway transit services in Algiers: priority improvement actions based on users perceptions. Transport Policy, v. 53, issue C, p. 175-185, 2017

MADL, T., FRANKLIN, S., KE CHEN, TRAPPL, R., and MONTALDI, D. Exploring the structure of spatial representations. PLOS One, v. 11, n. 6, 2016.

MAY, A., PARKER, C. J., TAYLOR, N., ROSS, T. Evaluating a concept design of a crowd-sourced 'mashup' providing ease-of-access information for people with limited mobility. Transportation Research Part C: Emerging Technologies, v. 49, p.103-113, 2014.

MESHUR, H., 2013. Accessibility for People with Disabilities in Urban Spaces: A Case Study of Ankara, Turkey. Archnet-IJAR: International Journal of Architectural Research, vol. 7, issue 2, p. 43-60, 2013.

MINISTÉRIO DAS CIDADES. Manual de BRT - Bus Rapid Transit: guia de planejamento. Brasília: Institute for Transportation and Development Policy, 2008.

MONZON A., HERNANDEZ S., CASCAJO R. Quality of bus services performance: benefits of real time passenger information systems. Transport and Telecommunication Journal, v. 14, n. 2, p. 155-166, 2013. 


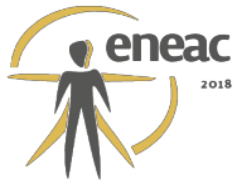

ROBERTS, M. J., GRAY, H., \& LESNIK, J. Preference versus performance: Investigating thedissociation between objective measures and subjective ratings of usability for schematic metro maps and intuitive theories of design. International Journal of Human-Computer Studies, v. 98, issue C, p. 109-128, 2016.

RODRIGUEZ-SANCHEZ, M. C., MARTINEZ-ROMO, J. GAWA Manager for accessibility Wayfinding apps, International Journal of Information Management. The Journal for Information Professionals, v.37, n.6, p.505-519, Dez. 2017.

SAMPAIO, R.F, MANCINI, M.C. Estudos de Revisão Sistemática: Um Guia para Síntese Criteriosa da Evidência Científica. Revista Brasileira de Fisioterapia. São Carlos, v.11, n.1, p 83-89, jan/fev. 2007

SCHMITT, L., G. CURRIE, DELBOSC, A. Measuring the Impact of Unfamiliar Transit Travel Using a University Access Survey. Transport Policy, v. 30, p. 301-307, 2013.

STOPKA, O., BARTUŠKA, L., KAMPF, R. Passengers' evaluation of the integrated transport systems. Nase More, Dubrovnik, v. 62, p. 153-157, 2015.

SUSILO, Y., CATS, O. Exploring key determinants of travel satisfaction for multi-modal trips by different traveler groups. Transportation Research Part A: Policy and Practice, v. 67, issue C, p. 366-380, 2014.

TUOMINEN, A.; JÄRVI, T.; RÄSÄNEN, J.; SIRKIÄ A. \& HIMANEN, V. (2007). Common preferences of different user segments as basis for intelligent transport system: case study - Finland. IET Intelligent Transport Systems, v. 1, n. 2, p. 59-68, 2007.

TYRINOPOULOS, Y., ANTONIOU, C. Factors Affecting Modal Choice in Urban Mobility. European Transport Research Review, v. 5, n. 1, p. 27-39, 2013

VAN DER HOEVEN, F., \& VAN NES, A. Improving the design of urban underground space in metro stations using the space syntax methodology. Tunnelling and Underground Space Technology, 40, p. $64-74$, fev. 2014.

WESTERHEIM, H., HAUGSET, B., and NATVIG, M. Developing a unified set of information covering accessibility at public transport terminals'. IET - Intelligent Transport Systems, vol.1, n. 2, p. 75-80, 2017.

WRIGHT, C. Facilitando o transporte para todos. $1^{\frac{a}{a}}$ ed. Washington, D.C. :Banco Interamericano de Desenvolvimento, 2001. 